\title{
Closed-Loop Radar with Adaptively Matched Waveforms
}

\author{
Nathan A. Goodman \\ Department of Electrical and Computer Engineering, University of Arizona \\ 1230 E. Speedway Blvd, Tucson, Arizona 85721 \\ goodman@ece.arizona.edu
}

\begin{abstract}
Adaptive and knowledge-based radar focus on improving the performance of the radar receiver through signal processing. However, rather than develop transmission waveforms and signal-processing techniques independently, it is useful to consider a closed-loop system complete with an adaptive radar transmitter.
\end{abstract}

In this paper, we summarize and demonstrate a framework being developed at the University of Arizona for implementation of closed-loop radar with adaptive waveforms. This framework integrates a Bayesian channel representation, matched illumination techniques, and sequential hypothesis testing. The result is a closed-loop system that modifies its understanding of the channel based on measured data, customizes waveforms to hasten understanding of the channel, and draws conclusions (such as target classification) when sufficient understanding of the propagation channel is achieved.

\section{INTRODUCTION}

Adaptive and knowledge-based (KB) signal processing focus on improving radar performance through advanced signal processing at the receive end of the system. However, rather than develop transmission waveforms and signalprocessing techniques independently, it is useful to consider the implementation and performance of a closed-loop radar system. The closed-loop system possesses an adaptive radar transmitter that responds to the propagation environment and to previously received data. This type of system, termed cognitive radar in [1], can be viewed as an intelligent system that continually interrogates the environment in order to achieve its objectives.

In related work, Bell applied information theory to design radar waveforms in [2]. In [3], Guerci and Pillai develop a theory for the two-target ID problem and then propose an extension to the $M$-target ID problem. The problem of temporal waveform shaping for improved target detection and identification is also considered in [4].

In [5], we have presented a closed-loop framework for radar target identification when the targets are each characterized by a known impulse response. In [5], we used sequential hypothesis testing to determine when the experiment should be terminated while achieving a desired error rate. Two different matched waveform techniques were compared.
In this paper, we begin by summarizing the cognitive radar framework being developed at the University of Arizona. This framework integrates a Bayesian channel representation, matched illumination techniques, and sequential hypothesis testing. The result is a closed-loop system that modifies its understanding of the channel based on measured data, customizes waveforms to hasten understanding of the channel, and draws conclusions (such as target detection or classification) when sufficient understanding of the propagation channel is achieved. We then demonstrate the effectiveness of our framework with simulated results from two applications. The first application is a target recognition scenario while the second uses the closed-loop framework to control transmit beamforming for integrated search and track.

\section{Closed-Loop, OR Cognitive, RADAR}

At the University of Arizona, we are researching capabilities and implementations of the Bayesian approach to cognitive radar described in [1]. In this approach, the radar is responsible for deriving various hypothetical alternatives that could describe the radar channel. Since prior knowledge and data can be inaccurate, corrupt, or outdated, the receiver also assigns probabilities to these hypotheses and feeds the information back to the transmitter. The transmitter is responsible for minimizing the uncertainty associated with the competing hypotheses in light of other restrictions such as priorities placed on certain aspects of the hypotheses. For example, the transmitter may choose to minimize uncertainty about potentially threatening targets while sacrificing uncertainty about non-threatening targets. The transmitter can minimize uncertainty through waveform design, platform control, and collaboration with other sensors.

To apply the Bayesian approach, it is necessary to have an efficient description of the channel. This description contains information about the channel such as target tracks, clutter, and external interference. Moreover, the description can be converted into impulse responses and statistical information about the interference. In other words, the channel description contains an encoding of the information necessary for describing expected components of the received signal. Uncertainty about the channel state manifests itself as a probability density function. The adaptive illuminator must evaluate the ensemble of channel states inherent in the description and determine a waveform 
to transmit that minimizes the uncertainty about the channel at the next interval. In other words, we want to customize the system's interrogation of the channel in order to minimize uncertainty about the channel. Formulation of this problem in much more detail, however, is applicationspecific and is an important component of our ongoing research.

Evaluation of the channel state uncertainty after a data collection requires knowledge of the state's pdf given the data. This relationship can be described with Bayes' Theorem as

$$
p\left(\mathbf{x}(n+1) \mid r_{n}(t)\right)=\frac{p\left(r_{n}(t) \mid \mathbf{x}(n)\right) p(\mathbf{x}(n))}{p\left(r_{n}(t)\right)}
$$

where $\mathbf{x}(n)$ is the channel state descriptor at time step $n$ and $r_{n}(t)$ is the received waveform due to the active illumination at time step $n$. The denominator term in (1) is a scale factor common to all channel alternatives, and since the channel descriptor can be used to describe the deterministic and random components of $r_{n}(t)$, there is hope that the likelihood $p\left(r_{n}(t) \mid \mathbf{x}(n)\right)$ can be evaluated. The remaining component, $p(\mathbf{x}(n))$, is obtained from the probabilistic rating of the channel prior to data collection and includes propagation of time-varying channel components up to the time instant of the data collection. Just as in traditional target tracking, longer time intervals between state predictions lead to larger uncertainty.

Target parameters such as range and Doppler shift are continuous. Therefore, there is an infinite span of potential channel states that must be updated using (1). In order to make the problem practical, the resolution of the system in range, Doppler, and angle must be used to limit the number of channel states being considered.

In Fig. 1, the Bayesian framework for cognitive radar is compared to human perception and decision-making. In Fig. 1a, cognitive radar interrogates its environment, processes the data and other knowledge sources into an ensemble of channel hypotheses, then feeds the information back to the transmitter. The transmitter makes decisions about which platform should move, which should transmit, and what should be transmitted. In Fig. 1b, a human observes the environment through the five senses. The observations are assimilated into an understanding of the environment. Then, decisions can be made, and part of the decision-making process is whether or not to optimize the use of one of the senses.

The closed-loop radar interrogation procedure is as follows. At each iteration, we transmit a waveform matched to the probabilistic rating of target alternatives and collect the resulting data. Once we have the data, we form likelihood ratios and update our understanding of the channel in order to optimize the next waveform. While the radar continues to

\section{Cognitive Radar}

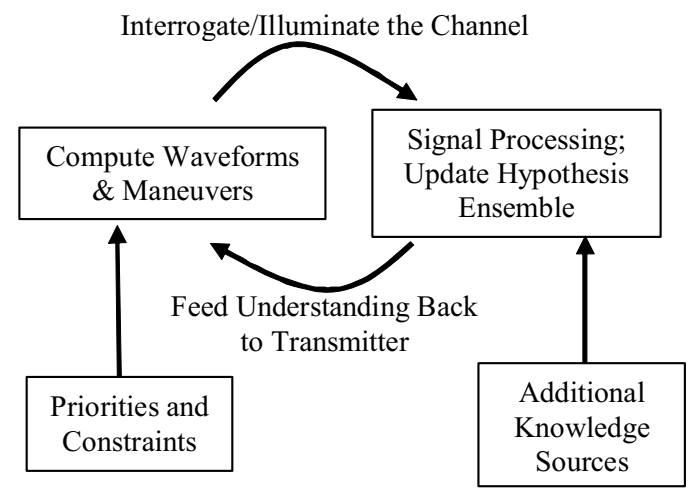

(a)

\section{Perception and Decision-Making}

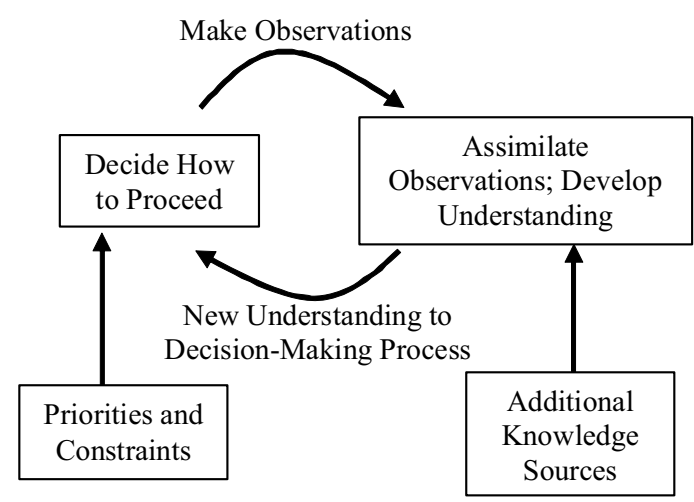

(b)

Figure 1. Block-diagram comparison of Bayesian-based cognitive radar to perception and decision making.

operate in this closed-loop fashion indefinitely, it is also important for the radar user to be able to draw traditional conclusions about the channel such as detections, tracks, and identifications. Therefore, at each iteration we can also compare the ensemble probabilities to thresholds derived from the theory of sequential hypothesis testing. If one of the hypotheses meets the threshold, a concrete decision can be made. Whether or not conclusions can be drawn from the channel, the interrogation of the channel continues. The goal of cognitive radar is generally to make conclusions more quickly and over a wider area with fewer resources.

\section{ADAPTIVE WAVEFORMS FOR TARGET RECOGNITION}

The first demonstration application that we consider is the target recognition scenario reported in [5]. We assume that a target is known to be present within the radar channel and that the target is known to be one of $M$ alternatives characterized by a known impulse response $h_{i}(t), i=$ $1,2, \ldots, M$. When the waveform $s(t)$ is transmitted, the received signal can be represented as 


$$
y(t)=h(t) * s(t)+n(t)
$$

where $*$ denotes the convolution operator, $y(t)$ is the received signal, and $n(t)$ is additive white Gaussian noise (AWGN) with average power normalized to $\sigma_{n}^{2}=1$.

Next, we let $\alpha_{i, j}$ for $i \neq j$ be the desired probability of incorrectly selecting $H_{j}$ given that $H_{i}$ is true. In sequential hypothesis testing, the experiment is terminated and $H_{m}$ is selected when the condition

$$
\Lambda_{m, j}^{k}>\frac{1-\alpha_{m, j}}{\alpha_{m, j}} \text { for all } j \neq m
$$

is met for some $m$ where $\Lambda_{m, j}^{k}$ is the likelihood ratio test for the $m^{\text {th }}$ and $j^{\text {th }}$ hypotheses after the $k^{\text {th }}$ data collection.

To compute (3), the pdf of the data under each hypothesis is needed. According to (2), the observation waveform at each iteration is a Gaussian random process with mean determined by the true impulse response and the actual transmit waveform. Hence, the pdf of the resulting (sampled) data vector $\mathbf{y}$ is multivariate Gaussian. The mean of $\mathbf{y}$ varies for each hypothesis and also as the transmit waveform adapts according to closed-loop operation. In this scenario, however, since the transmit waveform and target impulse responses are known, the pdf of the data is known under each hypothesis.

We apply two different matched waveform techniques. The first technique is based on maximizing mutual information (via waterfilling) as originally described in [2]. The second technique is based on SNR considerations as described in [3-4]. The application of both techniques to this application has been summarized in [5].

In Fig. 2, we show results from simulating this application with four target hypotheses. We generated 1500 different sets of four target impulse responses by generating sample functions of a Gaussian random process. Once the impulse responses were generated, it was assumed that they were known perfectly. The specified error rate was set at 0.01 . For each set of impulse responses, we continued the closed-loop procedure until the sequential testing threshold was crossed. We then noted the number of illuminations required for making a decision, and the final metric shown is the number of illuminations averaged over all trials. Fig. 2 indicates that for this application the SNR-based matched illumination technique with closed-loop adaptive matching is the best performer, though both matched illumination techniques outperform the non-adaptive wideband waveform and non-adaptive matched waveforms.

Next, we reconsider the target recognition application by allowing each target hypothesis to be represented by a power spectral density (PSD) rather than a single known impulse response. This modification affects the closed-loop

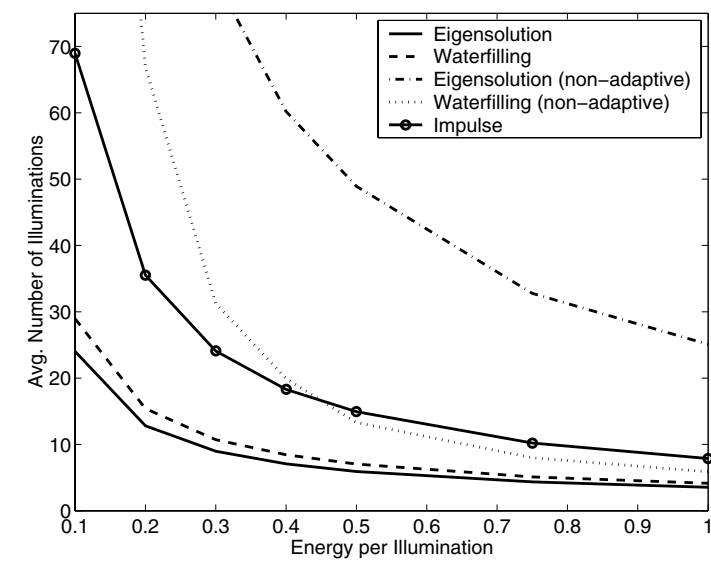

Figure 2. Average iterations to make a target ID decision.

procedure in two different ways. First, the waveform matching techniques must be extended to account for the different manner of representing the hypotheses. For example, the information-based matched illumination technique requires definition of a spectral variance function. This function describes the variance between the transfer functions of the hypotheses and is used with the waterfilling procedure to define the waveform spectra. In the results presented here, we define the spectral variance as

$$
\sigma_{H}^{2}(f)=\sum_{i=1}^{M} \operatorname{Pr}\left(H_{i}\right) \Psi_{i}(f)-\left|\sum_{i=1}^{M} \operatorname{Pr}\left(H_{i}\right) \sqrt{\Psi_{i}(f)}\right|^{2}
$$

where $\Psi_{i}(f)$ is the PSD of the $i^{\text {th }}$ hypothesis.

The second way in which statistically described hypotheses affect the closed-loop procedure is that it can be difficult to achieve the desired error rate when realizations of the different hypotheses are not mutually exclusive. To handle this, we defined the class PSDs such that most of their energy was in non-overlapping bands. This minimized the number of target realizations, or sample functions, whose frequency spectrum actually resembled the PSD of an incorrect target class more than the PSD of the correct target class. For realistic targets, non-mutually exclusive target classes is a common difficulty that must be accounted for.

Results for statistically described hypotheses are shown in Fig. 3 where we compare a non-adaptive wideband waveform, a non-adaptive matched waveform that is matched using only the initial target class probabilities, and an adaptive matched waveform that updates as the target class probabilities are updated. In Fig. 3, we show the CDF of the iterations required to make a decision. Note that the adaptive waterfilling approach usually requires fewer iterations than non-adaptive approaches, but can occasionally require a huge number of iterations. This behavior was not apparent in the case of known impulse responses. The matched waveforms in Fig. 3 are based on the waterfilling approach. 


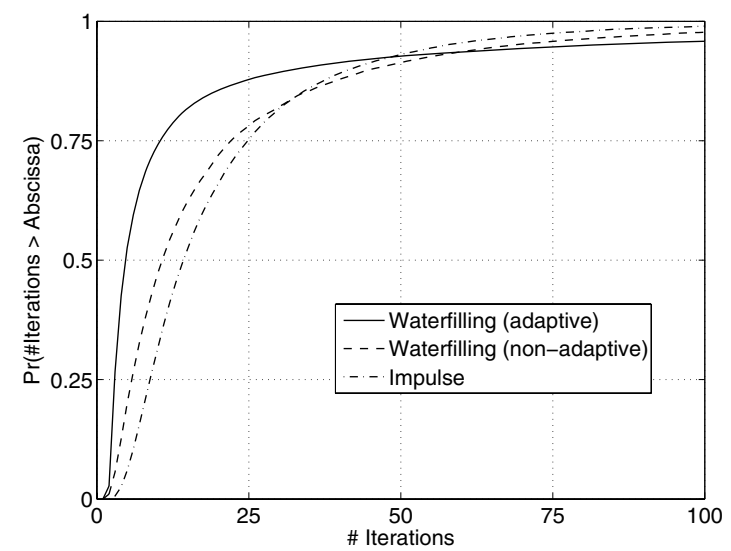

Figure 3. CDF of iterations required to make a target ID decision for statistically described targets.

\section{ADAPTIVE TRANSMIT BEAMFORMING}

Next, consider using the closed-loop framework for controlling an illumination beampattern. We assume that a volume of space can be divided into $M$ cells within which we will detect and track targets. Let the received data consist of the measurements made by an antenna array (we assume no range-Doppler resolution for the moment). According to the typical narrowband signal model for antenna arrays, the received data vector is

$$
\mathbf{y}=\sum_{i=1}^{N} S\left(k_{x}^{(i)}, k_{y}^{(i)}\right) \alpha_{i} \mathbf{v}\left(k_{x}^{(i)}, k_{y}^{(i)}\right)+\mathbf{n}
$$

where $S\left(k_{x}^{(i)}, k_{y}^{(i)}\right)$ is the illumination pattern voltage in the cell containing the $i^{\text {th }}$ target, $\alpha_{i}$ is the $i^{\text {th }}$ target's reflection coefficient, and $\mathbf{v}\left(k_{x}^{(i)}, k_{y}^{(i)}\right)$ is the $i^{\text {th }}$ target's steering vector, which depends on which cell the target is located through the spatial frequency coordinates $\left(k_{x}^{(i)}, k_{y}^{(i)}\right)$. The ability to detect a target in the $i^{\text {th }}$ cell depends on the SNR, which in turn depends on the illumination of the cell. Assuming a finite energy constraint, we are interested in optimally allocating energy to the resolution cells such that we can detect and track targets in the cells as energy-efficiently as possible. It must be noted here that this problem is essentially the same as the one treated in [6]; however, here we emphasize that the solution is obtained with the same framework used in Section III.

Consistent with the approach used for target identification, we can define a spectral variance function, $\sigma_{H}^{2}\left(k_{x}, k_{y}\right)$ for use with the waterfilling operation. Each cell, due to its different orientation with respect to the receive array, is associated with different spatial frequencies. The spectral variance associated with each spatial frequency cell depends on the expected target power as well as the probability of a target being present.

Fig. 4 shows a snapshot of the closed-loop beamforming procedure. On the left in Fig. 4, we show an image
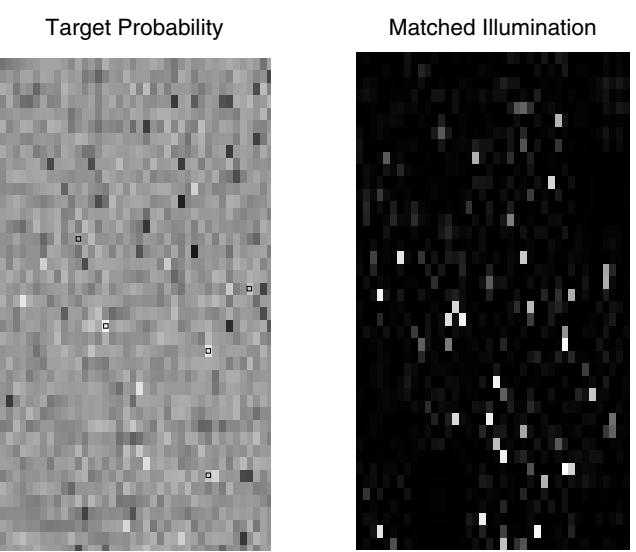

Figure 4. Target probability (left) and resulting matched illumination pattern (right). White is highest intensity.

representing the probability of target presence at one instant in time (true target locations shown with small squares). On the right, we show the spatial power pattern obtained from converting the probabilities on the left to a spectral variance function. Note that the most energy is allocated to cells where target presence is most uncertain.

\section{CONCLUSIONS}

We have presented a brief summary of ongoing research in closed-loop, or cognitive, radar at the University of Arizona. We are developing techniques for representing radar channels using a probabilistic methodology that is the foundation for closed-loop radar operation. We are also investigating techniques for converting such a probabilistic channel representation into matched waveforms that can be implemented with an adaptive transmitter. Two applications have been quickly demonstrated - one that implements temporal matching waveforms and one that implements spatially matched waveforms. In the future, we will combine these techniques to develop closed-loop radar systems that perform integrated search, track, and identification functions.

\section{REFERENCES}

[1] S. Haykin, "Cognitive radar: a way of the future," IEEE Sig. Proc. Mag., vol. 23, no. 1, pp. 30-40, Jan. 2006.

[2] M.R. Bell, "Information theory and radar waveform design," IEEE Trans. Info. Theory, vol. 39, no. 5, pp. 1578-1579, Sept. 1993.

[3] J.R. Guerci and S. U. Pillai, "Theory and Application of Optimum Transmit-Receive Radar," IEEE 2000 International Radar Conference, Washington, DC, pp. 705-710, May 8-12, 2000.

[4] D.A. Garren, M.K. Osborn, A.C. Odom, J.S. Goldstein, S.U. Pillai, and J.R. Guerci, "Enhanced target detection and identification via optimised radar transmission pulse shape," IEEE Proc. Radar, Sonar and Navigation, 148(3), pp. 130-138, June 2001.

[5] N.A. Goodman, P.R. Venkata, and M.A. Neifeld, "Adaptive waveform design and sequential hypothesis testing for target recognition with active sensors," IEEE J. Sel. Topics in Sig. Proc, vol. 1, no. 1, pp. 105-113, June 2007.

[6] D.R. Fuhrmann, "Active-testing surveillance systems, or, playing twenty questions with a radar," in Proc. 11th Annual Adaptive Sensor and Array Processing (ASAP) Workshop, MIT Lincoln Laboratory, Lexington, MA, Mar. 11-13, 2003 [Cd-Rom] 